\title{
OPTIMAL DESIGN OF REAL AND COMPLEX MINIMUM PHASE DIGITAL FIR FILTERS
}

\author{
Niranjan Damera-Venkata and Brian L. Evans \\ Embedded Signal Processing Laboratory, Dept. of Electrical and Computer Engineering \\ The University of Texas at Austin, Austin, TX 78712-1084 USA \\ \{damera-v,bevans\}@ece.utexas.edu
}

\begin{abstract}
We present a generalized optimal minimum phase digital FIR filter design algorithm that supports (1) arbitrary magnitude response specifications, (2) high coefficient accuracy, and (3) real and complex filters. The algorithm uses the Discrete Hilbert Transform relationship between the magnitude spectrum of a causal real sequence and its minimum phase delay phase spectrum given by Cizek. We extend the transform pair to the complex case. We show that the algorithm gives arbitrary coefficient accuracy. We present design examples that exceed the coefficient accuracy of the optimal real minimum phase filters reported by Chen and Parks and reduce the length of the optimal complex linear phase filters designed by Karam and McClellan.
\end{abstract}

\section{INTRODUCTION}

Given the shortest minimum phase and linear phase digital FIR filters that meet the same magnitude specification, e.g. optimal filters in the Chebyshev sense, the minimum phase filter would have two key advantages [1]:

1. reduced filter length that is one-half to three-fourths of the linear phase filter length [2], and

2. minimum group delay that concentrates energy in the low-delay instead of the medium-delay coefficients [3].

Minimum phase filters can simultaneously meet delay and magnitude response constraints yet generally require fewer computations and less memory than linear phase filters.

Previous algorithms for designing minimum phase digital FIR filters have been limited to real filters and may be divided into two classes. One class $[1,4,5,6,7]$ uses the following steps: (1) design an optimal linear phase FIR filter for a power spectrum that is a squared ideal piecewise constant magnitude response, (2) factor the linear phase polynomial transfer function (polynomial deflation), and (3) reconstruct the minimum phase filter coefficients (polynomial inflation). Polynomial inflation may introduce catastrophic numerical errors [15]. The second class $[8,9]$ deconvolves the complex cepstrum of the minimum phase filter impulse response. The Chen and Parks algorithm [7], which is based on root finding, produces the best coefficient accuracy among the reported design algorithms.

This research was supported by the US Defense Advanced Research Projects Agency under DARPA Grant DAAB07-97-CJ007, and a US NSF CAREER Award under grant MIP-9702707.
Complex digital FIR filters may have lower computational complexity vs. a real digital FIR filter for the same magnitude specification [10]. An algorithm to design optimal, complex, minimum phase filters has not been previously reported. One approach would be to extend the two classes of design algorithms for the real case. Factoring polynomials, however, becomes far less reliable because not every complex root will have a complex conjugate resulting in an ill-conditioned problem [11]. As in the real case, filter designs produced by root finding methods may suffer from numerical errors if a suboptimal polynomial inflation method is used. Extending the second class of algorithms requires a relation between the complex cepstrum and the minimum phase spectrum for complex sequences, which has not been previously reported.

In this paper, we present a robust non-iterative algorithm to design optimal minimum phase digital FIR filters with arbitrary magnitude responses based on the Discrete Hilbert Transform (DHT). Our algorithm is not affected by the location and number of zeros on or within the unit circle. It may be applied without modification for real and complex digital FIR filters. It controls the accuracy of the resulting minimum phase filter coefficients by a single parameterthe length of the FFT used to compute the DHT.

A significant benefit of our algorithm is that it can be extended to two dimensions by using the 2-D DHT [13]. In this case, we cannot guarantee optimality in the Chebyshev sense. If the magnitude response of the best non-minimum phase filter has no zeros on the unit bicircle, then we can produce a minimum phase filter with a virtually identical magnitude response, after application of the DHT [16].

Section 2 derives generalized DHT relationship for complex sequences. Section 3 describes the proposed design procedure. Section 4 provides two design examples using our algorithm. Section 5 gives conclusions and future research directions. The Appendix shows that the minimum FFT length is proportional to the number of stopband zeros of the linear phase filter and the desired accuracy in the minimum phase coefficients.

\section{DHT FOR COMPLEX SEQUENCES}

In this section, we derive a DHT relation between the magnitude spectrum of a causal complex sequence and its minimum group delay phase spectrum. We show that the functional form for the real case gives minimum group delay for 
the complex case also. When minimum phase delay is required, we must add an extra constant term to the phase response for the complex case.

Any sequence can be represented as a sum of conjugate symmetric and conjugate antisymmetric parts

$$
x[n]=x_{e}[n]+x_{o}[n]
$$

where

$$
\begin{aligned}
& x_{e}[n]=\frac{1}{2}\left(x[n]+x^{*}[-n]\right) \\
& x_{o}[n]=\frac{1}{2}\left(x[n]-x^{*}[-n]\right)
\end{aligned}
$$

such that $x^{*}$ represents the complex conjugate of $x$. Based on Fourier transform properties,

$$
\begin{array}{r}
x_{e}[n] \stackrel{\mathcal{F}}{\longleftrightarrow} X_{R}\left(e^{j \omega}\right) \\
x_{o}[n] \stackrel{\mathcal{F}}{\longleftrightarrow} j X_{I}\left(e^{j \omega}\right)
\end{array}
$$

where $X_{R}\left(e^{j \omega}\right)$ is the real part of $X\left(e^{j \omega}\right)$ and $X_{I}\left(e^{j \omega}\right)$ is the imaginary part of $X\left(e^{j \omega}\right)$. From (1),

$$
X\left(e^{j \omega}\right)=X_{R}\left(e^{j \omega}\right)+j X_{I}\left(e^{j \omega}\right)
$$

If $x[n]$ is causal, i.e. $x[n]=0$ for $n<0$, then it is possible to recover $x[n]$ from $x_{e}[n]$. Since the non-zero portions of $x[n]$ and $x[-n]$ do not overlap except at $n=0$, we have

$$
x[n]=2 x_{e}[n] u[n]-x^{*}[0] \delta[n]
$$

Taking the Fourier transform of (7), we obtain

$$
X\left(e^{j \omega}\right)=\frac{1}{\pi} \int_{\pi}^{\pi} X_{R}\left(e^{j \theta}\right) U\left(e^{j(\omega-\theta)}\right) d \theta-x^{*}[0]
$$

$U\left(e^{j \omega}\right)$ is the Fourier transform of the unit step sequence:

$$
U\left(e^{j \omega}\right)=\frac{1}{1-e^{-j \omega}}+\sum_{k=-\infty}^{\infty} \pi \delta(\omega-2 \pi k)
$$

Using $\frac{1}{\left(1-e^{-j \omega}\right)}=\frac{1}{2}-\frac{j}{2} \cot \left(\frac{\omega}{2}\right)$ we can rewrite (9) as

$$
U\left(e^{j \omega}\right)=\frac{1}{2}-\frac{j}{2} \cot \left(\frac{\omega}{2}\right)+\sum_{k=-\infty}^{\infty} \pi \delta(\omega-2 \pi k)
$$

Using (10), we can express (8) as

$$
\begin{aligned}
X\left(e^{j \omega}\right)= & X_{R}\left(e^{j \omega}\right)+j X_{I}\left(e^{j \omega}\right) \\
= & X_{R}\left(e^{j \omega}\right)+\frac{1}{2 \pi} \int_{-\pi}^{\pi} X_{R}\left(e^{j \theta}\right) d \theta- \\
& \frac{j}{2 \pi} \int_{-\pi}^{\pi} X_{R}\left(e^{j \theta}\right) \cot \left(\frac{\omega-\theta}{2}\right) d \theta- \\
& \Re e\left(x^{*}[0]\right)-j \Im m\left(x^{*}[0]\right)
\end{aligned}
$$

Equating real and imaginary parts in (11), and noting that

$$
\Re e\left(x^{*}[0]\right)=\frac{1}{2 \pi} \int_{-\pi}^{\pi} X_{R}\left(e^{j \theta}\right) d \theta
$$

we obtain the relationship

$$
X_{I}\left(e^{j \omega}\right)=-\frac{1}{2 \pi} \int_{-\pi}^{\pi} X_{R}\left(e^{j \theta}\right) \cot \left(\frac{\omega-\theta}{2}\right) d \theta-\Im m\left(x^{*}[0]\right)
$$

If we consider the DHT for the complex cepstrum of a complex sequence, we obtain

$$
\arg X\left(e^{j \omega}\right)=-\frac{1}{2 \pi} \int_{-\pi}^{\pi} \log \left|X\left(e^{j \theta}\right)\right| \cot \left(\frac{\omega-\theta}{2}\right) d \theta-K
$$

where $K=\Im m\left(\hat{x}^{*}[0]\right)$. The minimum phase spectrum of a complex sequence includes the extra constant term $K$. Since group delay is the derivative of the phase spectrum, the constant $K$ term will vanish if we require minimum group delay for the minimum phase filters. So, if we seek minimum group delay digital FIR filters, then the same DHT relation holds for complex sequences An FFT-based algorithm [12] can compute (14).

\section{OPTIMAL DESIGN ALGORITHM}

The optimal minimum phase filter is designed by transforming an optimal linear phase FIR filter into an optimal minimum phase filter in two steps:

1. obtain the magnitude response of the optimal magnitude minimum phase FIR filter, and

2. apply the DHT to the magnitude response in step 1 .

Several methods achieve the first step. We modify the procedure by Chen and Parks [7] which uses a two-level ripple specification. In order to handle multiple ripple levels over different bands, we may replace step 1 with the method of Boite and Leach [8] which uses a modified Parks-McClellan algorithm to allow the error to oscillate between 0 and $2 \delta_{k}$ instead of $-\delta_{k}$ to $+\delta_{k}$, where $\delta_{k}$ is the ripple in the $k^{t h}$ band. Thus, optimal minimum phase filters with least complexity may be obtained for arbitrary magnitude specifications over the passband and the stopband using the DHT.

Chen and Parks derived relations between the linear phase and the optimal minimum phase filter specifications [7]. The relations calculate the design specifications on the minimum phase filter from the specifications on the optimal linear phase filter. Section 3.1 develops the design formulas to perform the conversion of specifications in the other direction since we design the linear phase filter first [19]. Section 3.2 applies the DHT to the optimal linear phase filter to compute the minimum phase filter.

\subsection{Step 1: Minimum Phase Magnitude Response}

We obtain the squared magnitude response of optimal magnitude minimum phase FIR filter in three steps. First, we design a symmetric lowpass filter of length $2 N-1$ using the Parks-McClellan algorithm. Given the desired passband and stopband ripples of the optimal minimum phase filter are $\delta_{1}^{\prime}$ and $\delta_{2}^{\prime}$, respectively, we compute the passband and stopband ripples of the linear phase filter which are respectively denoted as $\delta_{1}$ and $\delta_{2}$. The order of the minimum phase filter that is finally obtained is $N$. This filter is not only minimum phase but also has optimal magnitude characteristics in the Chebyshev sense. The length $2 N-1$ linear phase filter is typically designed with the smallest number of taps that meets the linear phase specifications.

$$
\delta_{1}=\frac{4 \delta_{1}^{\prime}}{2+2 \delta_{1}^{\prime 2}-\delta_{2}^{\prime 2}}
$$




$$
\delta_{2}=\frac{\delta_{2}^{2}}{2+2 \delta_{1}^{\prime 2}-\delta_{2}^{\prime 2}}
$$

Thus, given the specifications on the optimal minimum phase filter, we can design the equivalent linear phase filter to meet passband and stopband specifications $\delta_{1}$ and $\delta_{2}$. The transfer function of the linear phase filter is

$$
H_{L}(z)=z^{-(N-1)} \sum_{k=1}^{2 N-1} h_{L}(k) z^{k-1}
$$

Second, we shift the transfer function by $\delta_{2}+\epsilon$. Theoretically, the DHT does not exist on the unit circle [17], but adding $\epsilon$ ensures that the DHT of the filter exists. The $\epsilon$ term may be chosen to be arbitrarily small so as not to affect the magnitude spectrum significantly. We use $\epsilon=10^{-10}$. Then, we have a nonnegative frequency response $H_{L}(z)+\delta_{2}$ if we ignore the relatively small $\epsilon$ term.

Third, we normalize $H_{L}(z)+\delta_{2}$ using SCAL [7]

$$
\begin{gathered}
H(z)=\left(H_{L}(z)+\delta_{2}\right) S C A L \\
S C A L=\frac{4}{\left(\sqrt{1+\delta_{1}+\delta_{2}}+\sqrt{1-\delta_{1}+\delta_{2}}\right)^{2}}
\end{gathered}
$$

This causes passband ripple to oscillate between $1+\delta_{1}^{\prime}$ and $1-\delta_{1}^{\prime}$. The magnitude squared response of the minimum phase filter is

$$
H(z)=H_{M}(z) H_{M}\left(z^{-1}\right)
$$

where $H_{M}(z)$ has the required magnitude response of the minimum phase spectral factor.

\subsection{Step 2: Apply the DHT}

We directly apply the DHT to $H_{M}(z)$, which is the squareroot response of $H(z)$. We reconstruct the minimum phase polynomial from the magnitude response and the computed minimum phase response [15]. For any magnitude response, the minimum phase filter of a given order is unique. The algorithm is idempotent for the same value of $\epsilon$ to within the limits of the precision of the arithmetic being used.

\section{DESIGN EXAMPLES}

\subsection{Example 1: Real 325-Tap Lowpass Filter}

To show that the algorithm can handle very long filters, we design a real lowpass FIR filter with passband edge $F_{p}=0.28$, stopband edge $F_{s}=0.3$, weighting function [14] $1: 5 \times 10^{5}, \delta_{1}=.001660$, and $\delta_{2}=3.3627 \times 10^{-9}$ (computed using (15) and (16)). The optimal linear phase filter has a length of $L=649$, and optimal minimum phase FIR filter has a length of $N=325$. The design values of the passband and stopband ripples are 0.000830 and $8.2008 \times 10^{-5}$. Fig. 1 (a) shows the magnitude response and group delay of the optimal minimum phase filter. We used an FFT length of $524288\left(2^{19}\right)$, and the algorithm took $60 \mathrm{sec}$ to run. It turns out than using an FFT length of $32678\left(2^{15}\right)$, which only requires $10 \mathrm{sec}$ to run, will also produce a design that meets specifications. In the DHT-based optimal design, the actual values were $\delta_{1}^{\prime}=0.000828$ and $\delta_{2}^{\prime}=8.1684 \times 10^{-5}$. The relative percentage error was $.24 \%$ in the passband and $.39 \%$ in the stopband, which are comparable with the figures of $.29 \%$ and $.21 \%$, respectively, in [7]. While the ripples in both bands of the DHT-based design are lower than the specified value, the ripples in the design in [7] are greater than the specified values. In this case, the DHT-based algorithm produced a more desirable magnitude response than what was reported in [7].

\subsection{Example 2: Complex 26-Tap Lowpass Filter}

One of the advantages of our design approach is that it may be applied to both real and complex tap FIR filters without any changes. As an example, we design a lowpass filter with passband edge $F_{P}=0.7$, stopband edge $F_{s}=0.8$, and weighting function $1: 1$. The Karam and McClellan algorithm [10] gives an optimal linear phase complex-tap filter with 50 taps. Using the DHT-based algorithm, we can design a minimum phase complex FIR filter with 26 taps to meet the specifications. We calculate $\delta_{1}=0.004268$ and $\delta_{2}=0.004297 \mathrm{using}(15)$ and (16). The design values of $\delta_{1}^{\prime}$ and $\delta_{2}^{\prime}$ are 0.002125 and 0.092510 , respectively. Fig. 2 shows the magnitude response and group delay of the optimal complex filter. In the DHT-based optimal design, the actual values were $\delta_{1}^{\prime}=0.002125$ and $\delta_{2}^{\prime}=0.092359$. The relative errors are $0 \%$ in the passband and $.16 \%$ in the stopband. The designed values meet the specifications.

\section{CONCLUSION}

We present a new algorithm for the design of optimal minimum phase digital FIR filters with arbitrary magnitude responses. The algorithm avoids factoring of polynomials, does not involve iterative procedures, and can design real and complex filters. We also present a generalized DHT pair relating the magnitude and minimum phase responses of causal complex sequences. We note that the generalized transform requires an additional constant phase term which vanishes for the case of real coefficients.

\section{APPENDIX}

We quantify the minimum phase filter coefficient accuracy as a function of FFT length used to calculate the DHT that computes (14). The issue of accuracy arises because we constrain the complex cepstrum to be causal, while in practice this condition is only approximately satisfied. In [15], we derive the following formula based on the causality of the complex cepstral sequence

$$
\epsilon=2 \frac{N_{s}}{N_{F F T}}
$$

where $\epsilon$ is the upper bound of the deviation of the complex cepstrum from ideal, $N_{s}$ is the number of stopband zeros of the minimum phase filter, and the $N_{F F T}$ is the FFT length used to compute the DHT. Using this formula, a designer can decide on the minimum FFT length for the desired accuracy in the computed minimum phase filter coefficients. We can make $\epsilon$ arbitrarily small by using a large enough FFT length. For a filter with 100 stopband zeros, $\epsilon=.0977$ when using a 2048-point FFT, and $\epsilon=3.8 \times 10^{-4}$ when using $2^{19}$ point FFTs. This bound is conservative [15]. 

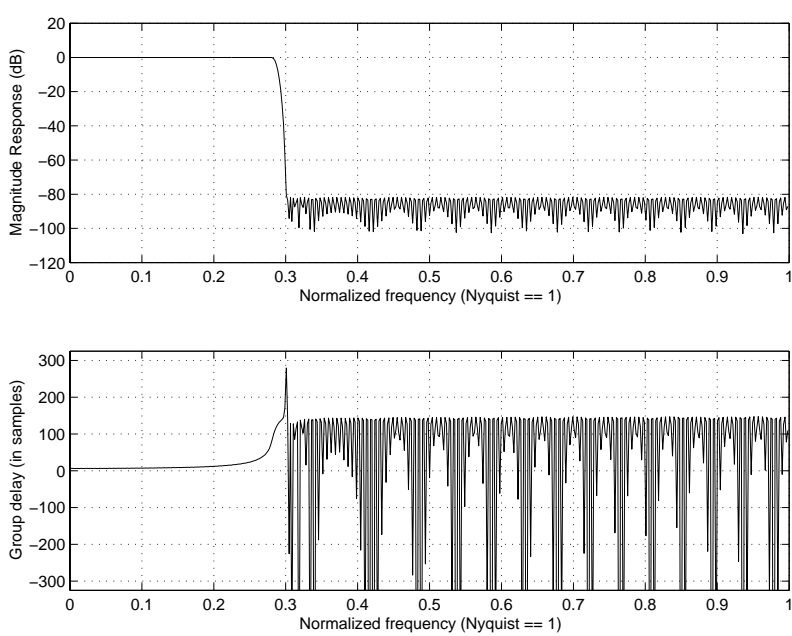

Figure 1: Magnitude response and group delay for an optimal real 325-tap minimum phase digital FIR filter designed by the new algorithm based on the DHT. The group delay for the optimal linear phase filter is 324 .

\section{ACKNOWLEDGEMENTS}

We would like to thank Mr. Shawn R. McCaslin, Prof. P. S. Reddy, and Prof. Dong Wei for their helpful suggestions.

\section{REFERENCES}

[1] O. Herrmann and H. W. Schüessler, "Design of nonrecursive digital filters with minimum phase," IEE Elect. Letters, vol. 6, no. 11, pp. 329-30, May 1970,

[2] P. Leistner and T. W. Parks, "On the design of FIR digital filters with optimum magnitude and minimum phase," Archiv fur Elektronik und Uebertragungstechnik, vol. 29, no. 6, pp. 270-4, Jun. 1975.

[3] E. A. Lee and David G. Messerschmitt, Digital Communication, 2nd ed., Kluwer, 1994.

[4] J. D. Fisher and T. W. Parks, "Optimal Chebyshev magnitude approximation for FIR Filters," IEEE Southwest. Conf., Houston, TX, Apr. 1973.

[5] Y. Kamp and C. J. Wellekens, "Optimal design of minimum phase FIR filters," IEEE Trans. Acoustics, Speech, Sig. Proc., vol. 31, no. 2, pp. 922-6, Aug. 1983.

[6] J. K. Liang and R. J. P. de Figueiredo, "A design algorithm for optimal lowpass non-linear phase digital filters," Sig. Proc., vol. 8, no. 1, pp. 3-21, Feb. 1985.

[7] X. Chen and T. W. Parks, "Design of optimal minimum phase FIR filters by direct factorization," Sig. Proc., vol. 10, no. 4, pp. 369-83, Jun. 1986.

[8] R. Boite and H. Leach, "A new procedure for the design of high order minimum phase FIR digital or CCD filters," Sig. Proc., vol. 3, no. 2, pp. 101-8, Apr. 1981.

[9] G. A. Mian and A. P. Nainer, "A fast procedure to design equiripple minimum phase FIR filters," IEEE
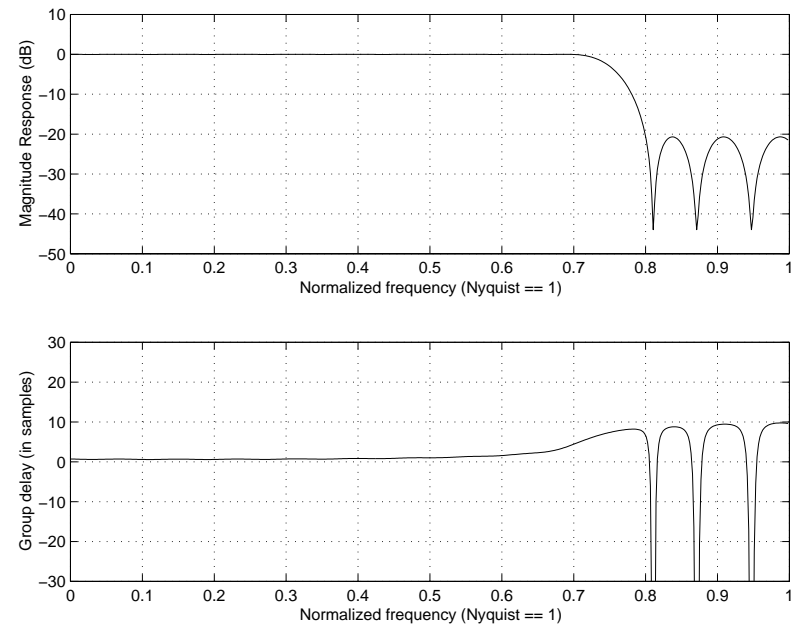

Figure 2: Magnitude response and group delay for an optimal complex 26-tap minimum phase digital FIR filter designed by the new algorithm based on the DHT. The group delay for the optimal linear phase filter is 25 .

Trans. Circuits and Sys., vol. 29, no. 5, pp. 327-31, May 1982.

[10] L. J. Karam and J. H. McClellan, "Complex Chebyshev approximation for FIR digital filter design," IEEE Trans. Circuits and Sys. II, vol. 42, no. 3, pp. 207-16, Mar. 1995.

[11] J. H. Wilkinson, Rounding Error in Algebraic Process, Prentice Hall, 1963.

[12] V. Cizek, "Discrete Hilbert Transform," IEEE Trans. Audio Electroacoust., vol. 18, no. 4, pp. 340-3, Dec. 1970.

[13] R. R. Read and S. Treitel, "The stabilization of twodimensional recursive filters via the Discrete Hilbert Transform," IEEE Trans. Geo Sci. Elect., vol. GE-11, pp. 153-60, Jul. 1973.

[14] T. W. Parks and J. H. McClellan, "Chebyshev Approximation for nonrecursive digital filters with linear phase," IEEE Trans. Circuit Theory, vol. 19, no. 2, pp. 189-94, Mar. 1972.

[15] N. Damera-Venkata, B. L. Evans and S. R. McCaslin, "Design of optimal minimum phase digital FIR filters using Discrete Hilbert Transform", IEEE Trans. Sig. Proc., submitted.

[16] N. Damera-Venkata, M. Venkataraman, M. S. Hrishikesh, and P. S. Reddy, "Stabilization of 2-D recursive digital filters by the DHT method," IEEE Trans. Circuits and Sys. II, accepted for publication.

[17] P. S. Reddy and H. S. N. Murthy, "Stabilization of 2-D digital filters via Hilbert Transform," IEE Elect. Letters, vol. 19, no. 2, pp. 68-9, Jan. 20, 1983.

[18] N. Damera-Venkata, Stabilization and Stability Testing of Multidimensional Recursive Digital Filters, B.S. Thesis, May 1997, Univ. of Madras, Madras, India.

[19] D. Wei, Personal Communication, Mar. 1, 1998. 